Check for updates

Cite this: RSC Adv., 2019, 9, 28399

\title{
Cold model investigation of mixing-separation time distribution in a multi-element process coupled cyclone reactor for ionic liquid-catalyzed isobutane/butene alkylation
}

\author{
Mingyang Zhang, (D) *a Xiaoyu Li, ${ }^{* b}$ Shijie $\mathrm{Li}^{\text {a }}{ }^{\text {Y Yan Gao, }}{ }^{\text {a }}$ Zhenbo Wang, (D) ${ }^{\mathrm{c}}$ \\ Linhua Zhang, ${ }^{a}$ Hao Zhang ${ }^{a}$ and Bin $\mathrm{Li}^{\mathrm{d}}$
}

The residence time distributions of a dispersed phase in a multi-element process coupled cyclone reactor for ionic liquid-catalyzed isobutane/butene alkylation were numerically studied with a CFD method. The Eulerian-Eulerian multiphase flow model and Reynolds stress model were applied to simulate the flow field distribution in the cyclone reactor. The time in which the dispersed phase flows from the inlet slot to the overflow outlet tube is defined as the mean mixing-separation time between two phases in the cyclone reactor. The effects of the structural parameter of the slot on the mean mixing-separation time were investigated in this work. The results show that the residence time distributions are unimodal except for the condition that the number of slots is 1 . Besides, it is concluded that the velocity of dispersed phase flow into the reaction chamber and the relative velocity between two phases in the cyclone reactor are the main influence factors. Based on the results, a prediction model to explain the relationship between the mean mixing-separation time and the velocity of two phases was established.

Received 5th July 2019

Accepted 20th August 2019

DOI: 10.1039/c9ra05088d

rsc.li/rsc-advances for alkylation, ionic liquids show a high reactivity and broad prospect for development. The articles by Yoo et al. show that ionic liquids are a promising substitute for $\mathrm{H}_{2} \mathrm{SO}_{4}$ and $\mathrm{HF}$ as the alkylation catalyst because of their safer operation, low consumption, and strong catalytic performance. ${ }^{8}$

Ionic liquid catalyzed isobutane/butene alkylation is a heterogeneous system wherein reactions occur in or near the interface between the ionic liquid and hydrocarbon phases. The intrinsic reaction rate is extremely fast due to the highly reactive carbonium intermediate. ${ }^{9}$ Therefore, the residence time of the ionic liquidhydrocarbon mixture is of fundamental importance to the alkylate quality. Thus, it is necessary to study and develop a matched reactor for ionic liquid-catalyzed isobutane/butene alkylation. A pioneering investigation of the novel reactor for the ionic liquidcatalyzed isobutane/butene alkylation was performed by Zhou et al. ${ }^{10}$ They proposed a loop reactor for the alkylation process. However, the ideal time to separate alkylate from the ionic liquid is unclear, which limits its industrial application.

Based on hereinbefore problems, we proposed a multi-element process coupled cyclone reactor for ionic liquid-catalyzed isobutane/butene alkylation. Cyclone reactor is developed from the cyclone and hydrocyclone, which can be used in the multi-phase reaction system with the aid of other equipment. Recently, many experts and scholars applied the cyclone and hydrocyclone as a reactor to the heterogeneous reaction system, such as wastewater treatment, ${ }^{11}$ pyrolysis processes, ${ }^{12}$ hydrogen production, ${ }^{13}$ dry
${ }^{a}$ School of Thermal Engineering, Shandong Jianzhu University, Jinan 250101,
Shandong, China. E-mail: zhangmingyang18@sdjzu.edu.cn
${ }^{b}$ College of Mechanical and Electronic Engineering, Shandong University of Science
and Technology, Qingdao, 266590, China. E-mail: lixy2018@sdust.edu.cn
${ }^{c}$ State Key Laboratory of Heavy Oil Processing, China University of Petroleum (East
China), 266580, Qingdao, China
${ }^{d}$ School of Energy and Power Engineering, Jiangsu University, Zhenjiang 212013, China 
scrubbing of $\mathbf{H C l},{ }^{14}$ fermentation processes, ${ }^{15}$ petroleum industry ${ }^{\mathbf{1 6}}$ etc. The works show that the cyclone reactor is a perspective subject.

Residence time refers to the time of the material flowing through an equipment. The work of Danckwerts was the first to introduce the concept of residence time distribution (RTD), ${ }^{17}$ presenting the flow and mixing of fluids in non-ideal tubes, mixers and reactors. The works on RTD in the hydrodynamic study of equipment were by the method of experiments and simulations. ${ }^{18,19}$ Gamba et al. simulated the RTD in a stirred tank by CFD software and validated the results of tracer method by experimental data and literature information. Adeosun and Lawal investigated the mixing characteristics in a T-junction microchannel using residence time distribution. They used the RTD and coefficient of variation to quantify the mixing behavior. Besides, RTD in a reactor is also an important parameter required for the design of reactor.

In our previous works, a cold-model system was established. The pioneering investigations of the mixing and separation characters in the cyclone reactor were conducted. In these works, the concentration distributions of dispersed phase were investigated. ${ }^{21}$ And a parameter, named dispersion uniformity was used to evaluate the dispersion performance of dispersed phase in the cyclone reactor. The recoveries of two phases were used to evaluate the separation performance of the cyclone reactor. ${ }^{22}$ Moreover, a numerical simulation method for flow field distribution of the cyclone reactor was established.,22,23 Besides, the droplet size distributions of dispersed phase were obtained by the focused beam reflectance measurement (FBRM D600L) and population balance model (PBM).

The aim of the present paper is to study the mean mixingseparation time between two phases in the cyclone reactor. In the cyclone reactor, the residence time distribution of dispersed phase is different from the mean mixing-separation time. The flow time of dispersed phase in the inlet cushion chamber and overflow outlet should be deducted from the residence time distribution to calculate the mean mixing-separation time. Based on this, the effects of the structure of dispersed phase inlet on the mean mixing-separation time were investigated. Moreover, an empirical equation for predicting the mean mixing-separation time in the cyclone reactor was established.

The paper is structured as follows. In Section 2, the structure and working principle of the multi-element process coupled cyclone reactor are introduced. In Section 3, the numerical method along with the governing equations and a brief description of the solver is discussed. The effects of the structural parameters on the mean mixing-separation time between two phases in the cyclone reactor are studied in Section 4. In Section 5, a simple empirical model to calculate the mean mixing-separation time under different conditions is suggested.

\section{Experiments}

\subsection{The cyclone reactor}

The basic structure of the mentioned cyclone reactor is shown in Fig. 1. The cyclone reactor is designed on the base of the axial-axial-flow hydrocyclone developed by Wang et al. ${ }^{24}$ The C4 hydrocarbon mixture (dispersed phase) is injected through the symmetric tangential slots (inlet 2,3 ), while the ionic liquid (continuous phase) flows axially from inlet 1 into the cyclone reactor. A guided vane lies below the continuous phase inlet. After entering the cyclone reactor, a layer of ionic liquid forms near the wall surface in the reaction chamber of cyclone reactor
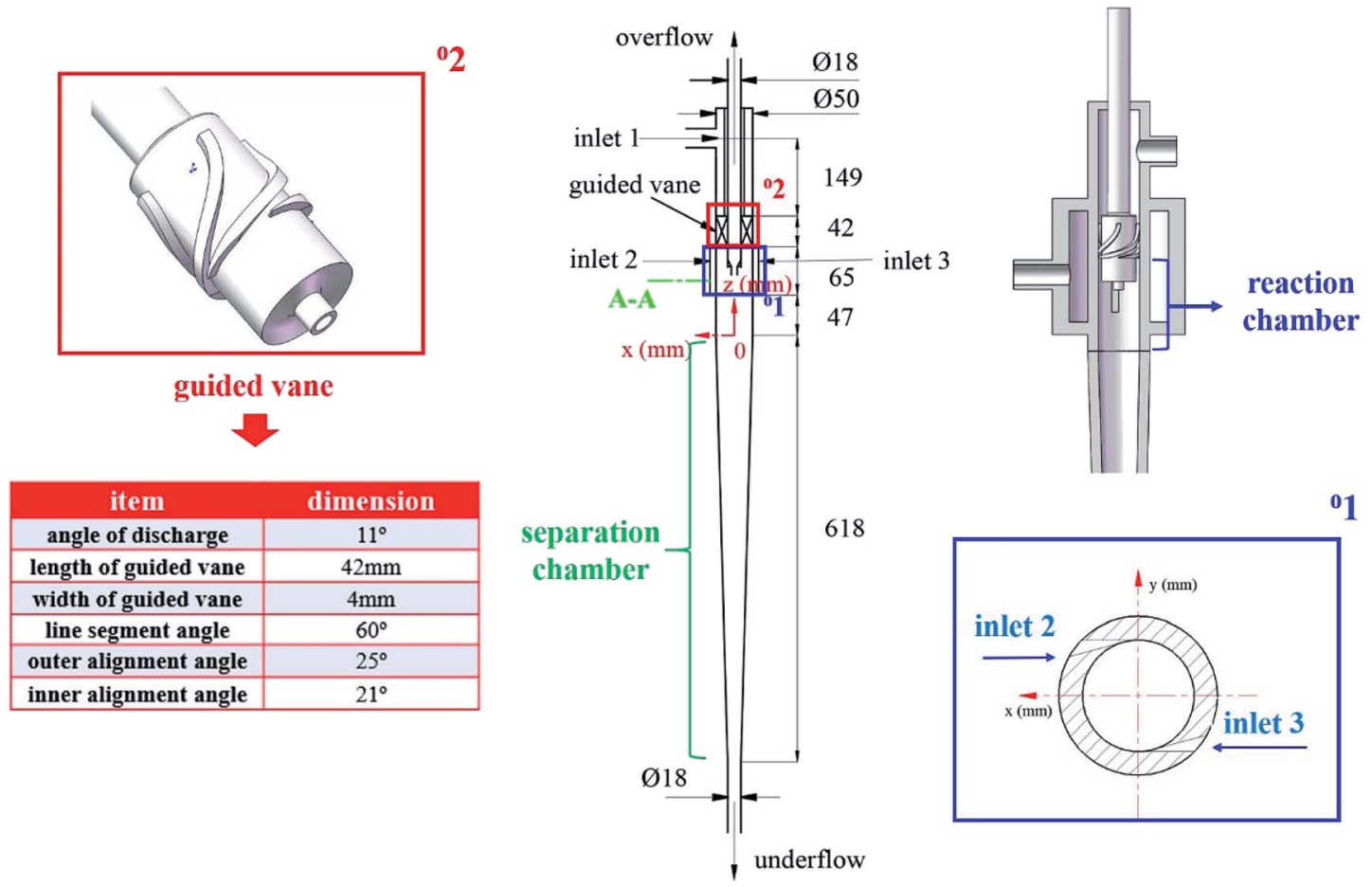

$\mathbf{0} 1$

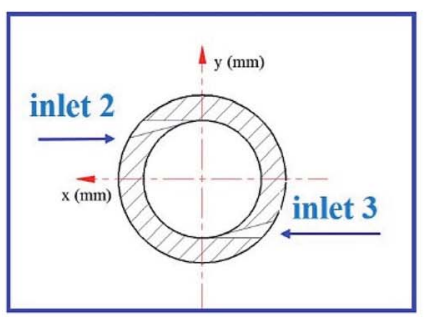

Fig. 1 Schematic diagram of the cyclone reactor structure. 
under the function of guided vane. Meanwhile, the $\mathrm{C} 4$ hydrocarbon mixture moves to the axis with the effect of centrifugal force after entering the LLCR through the tangential slots. ${ }^{25}$ During the moving process, the dispersed phase oscillates to liquid film, liquid film draws to form droplets and the droplets breakup under the action of shear force, which is the mixing process between two phases. Afterwards, the liquid mixture flows into the separation chamber. Under the influence of the structure of cyclone reactor and the density difference between reaction products and ionic liquids, the reaction products and ionic liquids discharges from the overflow outlet and underflow outlet, respectively. Namely, the cyclone reactor couples the mixing, reaction and separation element process. The integration of multi-element processes in a single equipment can effectively reduce the occurrence of side reactions, improve the yield of target products and simplify the process flow.

\subsection{The cold-model investigation system}

As mentioned above, the mixing, reaction and separation element processes occur in two phases throughout in the cyclone reactor. Therefore, the concentration distributions and droplet size distributions of dispersed phase obtained by the cold-model investigation system can be used to evaluate the mixing performance between two phases. Besides, the recoveries of two phases at outlets can be used to evaluate the separation efficiency of the cyclone reactor. Hence, with the consideration of cost, preliminary studies were conducted on the cold-model investigation system.

In the cold-model investigation system, kerosene and $74 \mathrm{wt} \%$ glycerin water solution were used as the cold-model test liquids, which were chosen for hydrocarbon-ionic liquid system owing to their density and viscosity are close to hydrocarbonionic liquid as shown in Table 1.

\subsection{The definition of structural parameters}

In this paper, four structural factors, i.e., height of the slit $(h)$, distance of the slit $(d)$, number of the slit $(n)$ and angle of the slit $(a)$ were studied, which can be seen in Fig. 2. The height of the slit was varied at $45,50,55,60,65,70 \mathrm{~mm}$. The distance of the slit was varied at $0.5,1.0,1.5,2.0,2.5,3.0 \mathrm{~mm}$. The number of the slit was varied to seven levels, i.e., 1, 2, 3, 4, 5, 6, 8. And the angle of the slit was adjusted to seven levels, which were $5^{\circ}, 7^{\circ}, 9^{\circ}, 11^{\circ}, 13^{\circ}, 15^{\circ}, 17^{\circ}$. Moreover, the effect of the total inlet flow on the mean mixingseparation time was used to verify the accuracy of the empirical model to calculate the mean mixing-separation time.

\section{Governing equations and flow solver}

Numerical simulations are performed on a two-phase liquidliquid system using the Eulerian multiphase model and Reynolds stress model (RSM), which are realized in the software Ansys15.0, and the liquids used are considered as incompressible fluids. The governing equations describing the flow are the conservation equations for mass and momentum. The description of multiphase flow as interpenetrating continua incorporates the concept of phasic volume fractions, denoted here by $\alpha_{\mathrm{q}}$. Volume fractions represent the space occupied by each phase, and the laws of conservation of mass and momentum are satisfied by each phase individually. The derivation of the conservation equations can be done by ensemble averaging the local instantaneous balance for each of the

Table 1 Physical parameters of the two phases

\begin{tabular}{lllll}
\hline Fluids & Phase & Density $/ \mathrm{kg} \mathrm{m}^{-3}$ & Viscosity/Pa s & Surface tension/N m \\
\hline 74 wt\% glycerin solution & Continous & 1164 & 0.03304 & 0.0223 \\
Kerosene & Dispersed & 754 & 0.0016 & 0.0128 \\
Ionic liquid & Continous & 1312 & 0.0314 & 0.002 \\
C4 hydrocarbon mixture & Dispersed & 600 &
\end{tabular}
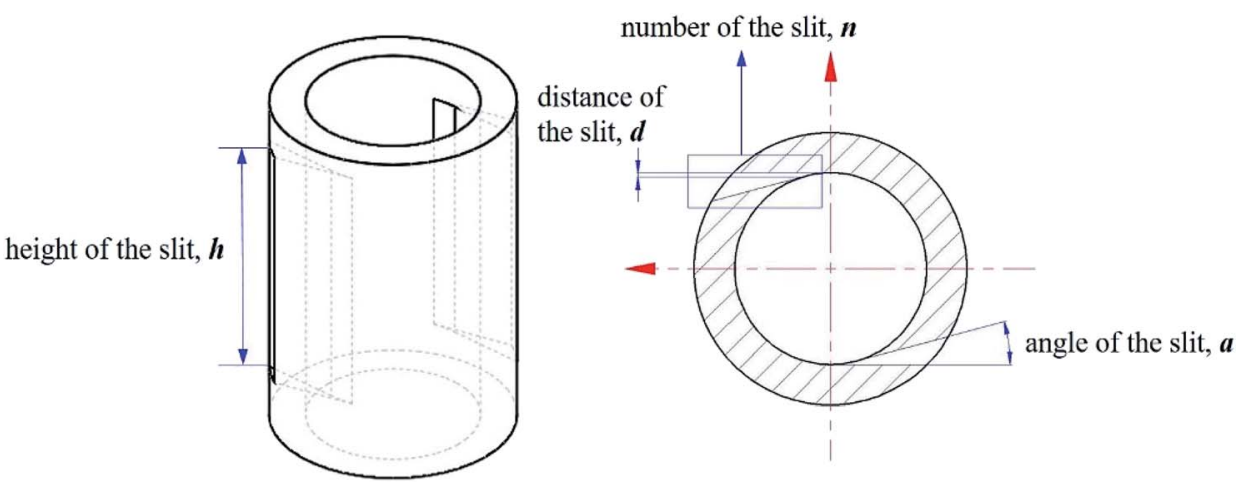

Fig. 2 The structural parameters under investigation. 
phases or by using the mixture theory approach. ${ }^{26,27}$ The continuity equation for phase $\mathrm{q}$ is,

$$
\frac{\partial}{\partial t}\left(\alpha_{\mathrm{q}} \rho_{\mathrm{q}}\right)+\nabla \cdot\left(\alpha_{\mathrm{q}} \rho_{\mathrm{q}} \vec{\nu}_{\mathrm{q}}\right)=0
$$

where $\vec{v}_{\mathrm{q}}, \rho_{\mathrm{q}}$ is the velocity and density of phase q.

The momentum balance for phase $q$ yields

$$
\begin{aligned}
& \frac{\partial}{\partial t}\left(\alpha_{\mathrm{q}} \rho_{\mathrm{q}} \vec{v}_{\mathrm{q}}\right)+\nabla \cdot\left(\alpha_{\mathrm{q}} \rho_{\mathrm{q}} \vec{v}_{\mathrm{q}} \vec{v}_{\mathrm{q}}\right) \\
& =-\alpha_{\mathrm{q}} \nabla p+\nabla \cdot \overline{\overline{t_{\mathrm{q}}}}+\alpha_{\mathrm{q}} \rho_{\mathrm{q}} \vec{g}+\sum_{\mathrm{p}=1}^{n}\left[K_{\mathrm{pq}}\left(\vec{v}_{\mathrm{p}}-\vec{v}_{\mathrm{q}}\right)\right]+\left(\vec{F}_{\text {lift, }}+\vec{F}_{\mathrm{Vm}, \mathrm{q}}\right)
\end{aligned}
$$

Here $\vec{v}_{\mathrm{p}}$ is the velocity of phase $\mathrm{p}, K_{\mathrm{pq}}$ is interphase exchange coefficients, $\vec{F}_{\text {lift,q }}$ is a lift force, the lift force acting on a secondary phase $\mathrm{p}$ in a primary phase $\mathrm{q}$ is computed from

$$
\vec{F}_{\text {lift }, \mathrm{q}}=-C_{\mathrm{l}} \rho_{\mathrm{q}} \alpha_{\mathrm{p}}\left(\vec{v}_{\mathrm{q}}-\vec{v}_{\mathrm{p}}\right) \times\left(\nabla \times \vec{v}_{\mathrm{q}}\right)
$$

where $C_{1}$ is the lift coefficient, the Tomiyama model was used to calculate the lift coefficient in this paper. ${ }^{28}$

$\vec{F}_{\text {Vm,q }}$ in eqn (2) is a virtual mass force. For multiphase flows, virtual mass effect is considered when the relative velocity exists between the secondary phase $\mathrm{p}$ and the primary phase $\mathrm{q}$, which can be expressed as: ${ }^{29}$

$$
\vec{F}_{\mathrm{Vm}, \mathrm{q}}=0.5 \alpha_{\mathrm{p}} \rho_{\mathrm{q}}\left(\frac{\mathrm{d}_{\mathrm{q}} \vec{v}_{\mathrm{q}}}{\mathrm{d} t}-\frac{\mathrm{d}_{\mathrm{p}} \vec{v}_{\mathrm{p}}}{\mathrm{d} t}\right)
$$

The term $\frac{\mathrm{d}_{\mathrm{q}}}{\mathrm{d} t}$ denotes the phase material time derivative of the form

$$
\frac{\mathrm{d}_{\mathrm{q}}(\varphi)}{\mathrm{d} t}=\frac{\partial(\varphi)}{\partial t}+\left(\vec{v}_{\mathrm{q}} \cdot \nabla\right) \varphi
$$

$\overline{\overline{t_{\mathrm{q}}}}$ in eqn (2) is the q phase stress-strain tensor, which can be expressed as:

$$
\overline{\overline{t_{\mathrm{q}}}}=\alpha_{\mathrm{q}} \mu_{\mathrm{q}}\left(\nabla \vec{v}_{\mathrm{q}}+\nabla \overrightarrow{\mathrm{v}}_{\mathrm{q}}^{T}\right)+\alpha_{\mathrm{q}}\left(\lambda_{\mathrm{q}}-\frac{2}{3} \mu_{\mathrm{q}}\right) \nabla \cdot \vec{v}_{\mathrm{q}} \overline{\bar{I}}
$$

where $\mu_{\mathrm{q}}$ and $\lambda_{\mathrm{q}}$ are the shear and bulk viscosity of phase $\mathrm{q}$.

Moreover, and the exchange coefficient was obtained with the following equation:

$$
K_{\mathrm{pq}}=\frac{\alpha_{\mathrm{q}} \alpha_{\mathrm{p}} \rho_{\mathrm{p}} f}{\tau_{\mathrm{p}}}
$$

where $f$ is the drag function and $\tau_{\mathrm{p}}$ is the "particulate relaxation time", defined as

$$
\begin{gathered}
\tau_{\mathrm{p}}=\frac{\rho_{\mathrm{p}} d_{\mathrm{p}}{ }^{2}}{18 \mu_{\mathrm{q}}} \\
f=\frac{C_{\mathrm{D}} \mathrm{Re}}{24} \\
C_{\mathrm{D}}=\left\{\begin{array}{ll}
24\left(1+0.15 \mathrm{Re}^{0.687}\right. \\
0.44 \quad \mathrm{Re}>1000
\end{array}\right) / \mathrm{Re} \quad \mathrm{Re} \leq 1000
\end{gathered}
$$

where $d_{\mathrm{p}}$ is the diameter of the droplets of phase $\mathrm{p}$, which were set based on the experimental data. ${ }^{30} \mathrm{Re}$ is the relative Reynolds number.
In this paper, the transport equation of tracer is used to calculate the mixing-separation time distribution. The transport equation of tracer concentration is as follows,

$$
\frac{\partial}{\partial t}\left(\rho Y_{\mathrm{i}}\right)+\nabla \cdot\left(\rho \vec{v} Y_{\mathrm{i}}\right)=\nabla\left(\left(\rho D_{\mathrm{i}, \mathrm{m}}+\frac{\mu_{\mathrm{t}}}{\mathrm{Sc}_{\mathrm{t}}}\right) \nabla Y_{\mathrm{i}}\right)
$$

where $Y_{\mathrm{i}}$ presents the local mass fraction of each species, $D_{\mathrm{i}, \mathrm{m}}$ is the mass diffusion coefficient for species $\mathrm{i}$ in the mixture, $\mu_{\mathrm{t}}$ is the turbulent viscosity, $\mathrm{Sc}_{\mathrm{t}}$ is the turbulent Schmidt number, the default $\mathrm{Sc}_{\mathrm{t}}$ is 0.7 .

The pulse tracing method was used in the residence time distribution simulation. 5\% tracer was added at the dispersed phase inlet in one time step. And the variation of tracer volume fraction $c(t)$ at different times at the detection locations were detected. Bases on the volume fraction distributions, the residence time distribution function $E(t)$ and the average residence time (MRT) of dispersed phase were obtained, which can be calculated as follows,

$$
\begin{gathered}
E(t)=\frac{c(t)}{\int_{0}^{\infty} c(t) \mathrm{d} t} \\
\int_{0}^{\infty} E(t) \mathrm{d} t=1 \\
\text { MRT }=\int_{0}^{\infty} t E(t) \mathrm{d} t
\end{gathered}
$$

As shown in Fig. 1, the structure of the cyclone reactor is relatively complex, particularly near the guided vane and overflow outlet. Therefore, the local mesh improvement procedure and hexahedral mesh priority principle were used to improve the mesh quality. A typical mesh of the cyclone reactor is shown in Fig. 3. To validate the numerical results, the grid dependence was first examined to minimize numerical uncertainty. The turbulent kinetic energy and axial distribution of dispersed phase concentration at the A-A cross section $(z=55 \mathrm{~mm})$ using different grid numbers is shown in Fig. 4. The results show that the simulation

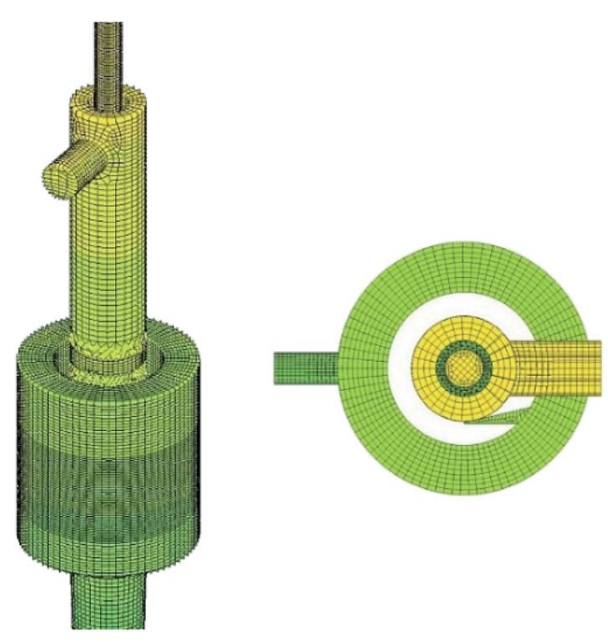

Fig. 3 Cyclone reactor meshes. 


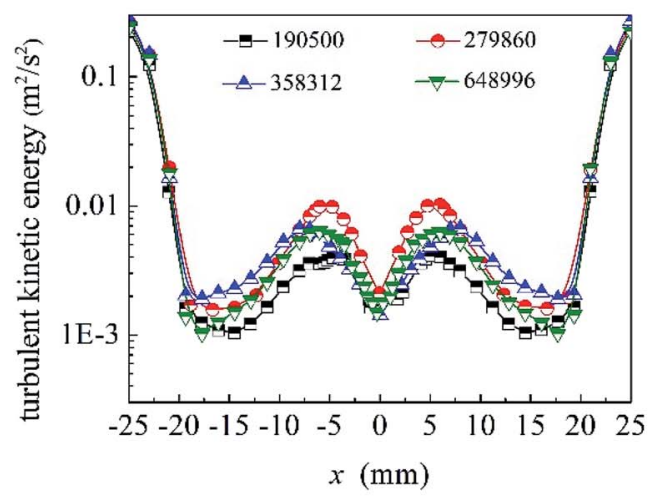

(a) turbulent kinetic energy

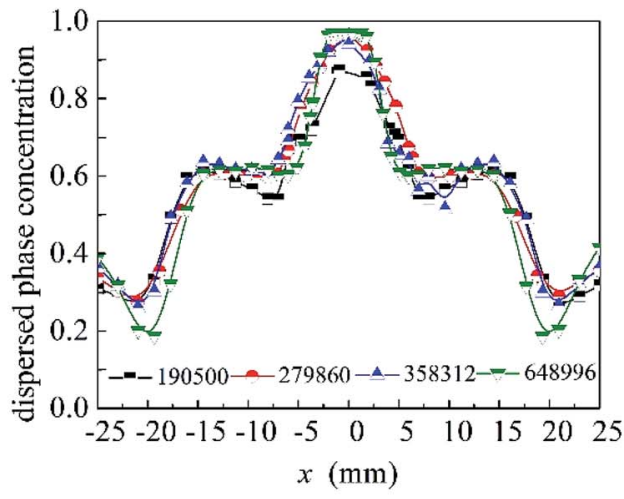

(b) dispersed phase concentration

Fig. 4 Grid independence verification.

results fitted well with the experimental data when the grid number was greater than 304400 . Therefore, a total of 304400 cells was used to maximize simulation cost and time.

\section{Results and discussions}

\subsection{Validity of the results}

The CFD method mentioned above was used to simulate the flow field in the cyclone reactor. And the line $x_{1} x_{2}$ at $z=$ $55 \mathrm{~mm}$ was set as the monitoring line, the coordinates of $x_{1}$ and $x_{2}$ are shown in Fig. 5 . To validate the simulation results, we compared the experimental data and the simulation results of the dispersed phase volume fraction $\left(\varepsilon_{\mathrm{d}}\right)$ at different radial position under different operational parameters (the overflow ratio, which is defined as the ratio of overflow outlet flow rate to the total inlet flow rate of two phases, is $0.4,0.5,0.6$, respectively). ${ }^{20}$ It can be seen from Fig. 5 that there is a good agreement between the experimental and predicted data and the error between them is less than $5 \%$. Therefore, the CFD method can be used to predict the flow field in the cyclone reactor.

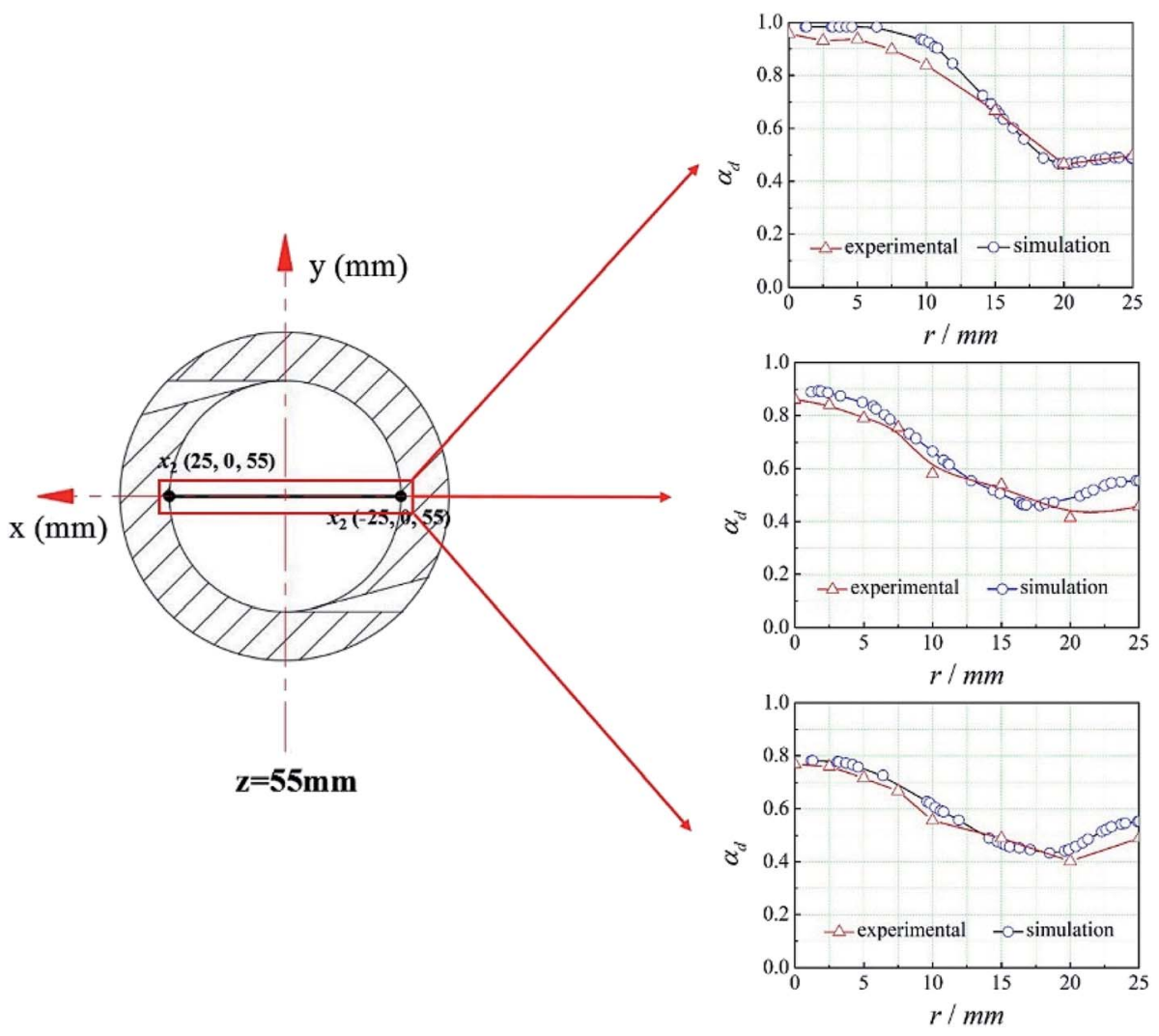

Fig. 5 Comparison between the experimental and predicted results. 

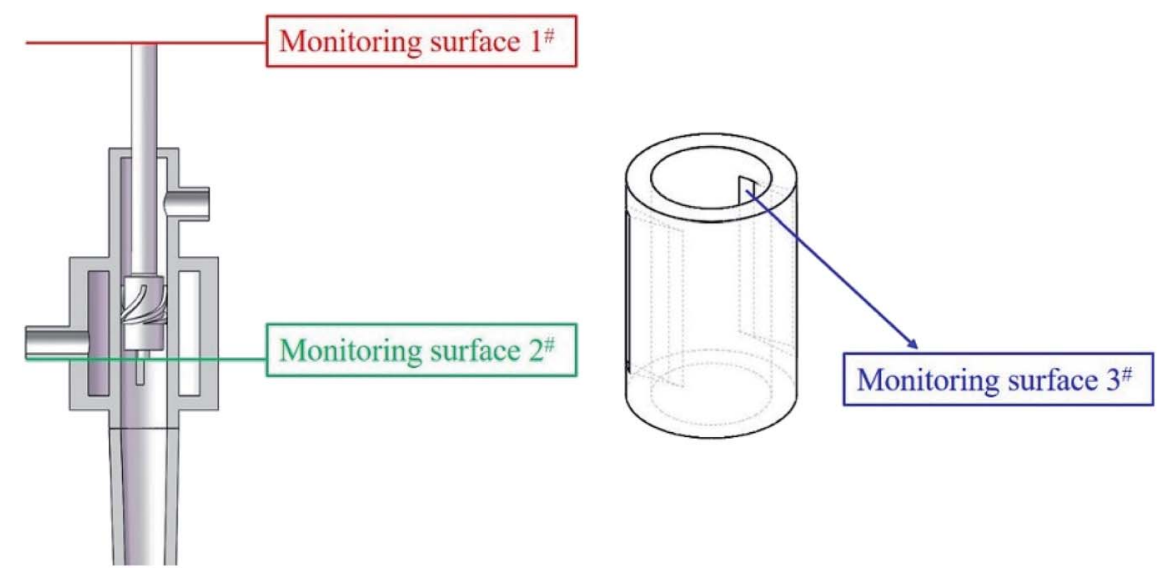

Fig. 6 Monitoring surface position.

\subsection{Defination of the mean mixing-separation time}

The positions of the three monitoring surface are shown in Fig. 6. As shown in Fig. 6, the MRT calculated on the $c(t)$ at monitoring surface $1^{\#}$ represents the residence time of dispersed phase in the cyclone reactor. However, the mixingseparation time between two phases in the cyclone reactor is different from the residence time of dispersed phase. The residence time in the cushion chamber and overflow tube should be ignored. Therefore, the flow time from monitoring surface $3^{\#}$ to monitoring surface $2^{\#}$ of dispersed phase is defined as the mixing-separation time between two phases in the cyclone reactor. Fig. 7 shows the difference between residence time distribution function $E(t)$ at three monitoring surfaces. As shown in Fig. 7, the RTD curves show one obvious peak with a tail, which indicates that dead-zone may exist in the cyclone reactor. Moreover, the peak of the residence time distribution at monitoring surface $3^{\#}$ is higher than the other two monitoring surfaces. And the position of the peak at monitoring surface $2^{\#}$ is on the left of the position of the peak at monitoring surface $1^{\#}$, which is consistent with that the time flow to monitoring surface $1^{\#}$ is longer then the time flow to monitoring surface $2^{\#}$.

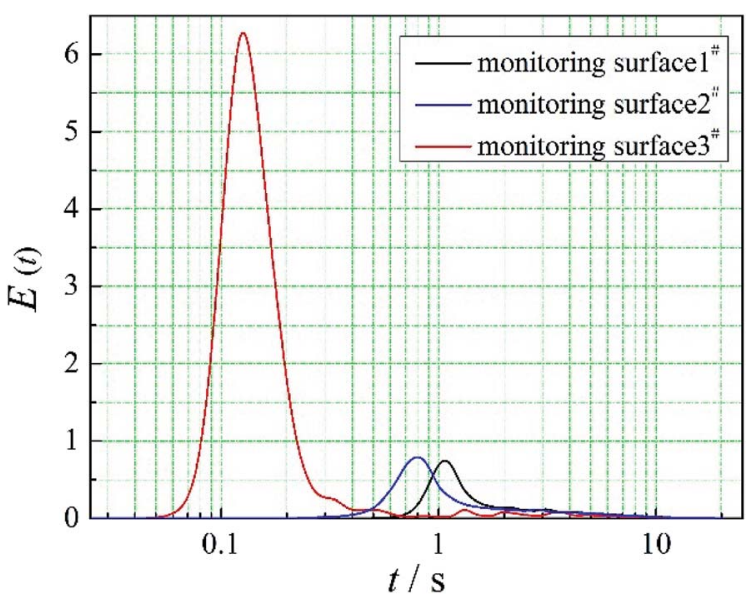

Fig. 7 RTD curves at monitoring surfaces.
4.3. Effect of structural parameters of the slot on the mixingseparation time between two phases

4.3.1. Effect of $a$ on the mixing-separation time between two phases. The effect of the angle of the slot on the RTD curves at monitoring surface $2^{\#}$ and $3^{\#}$ is shown in Fig. 8. As shown in Fig. 8, the variation of the RTD curves under different angles of the slot at monitoring surface $3^{\#}$ is more regular than that monitoring surface $2^{\#}$. With the increase of the angle of the slot, the change of the RTD curves is not obvious. Fig. 9 shows the effect of the angle of the slot on the mean mixing-separation time. As shown in Fig. 9, with the increase of the angle of the slot, the mixing-separation time between two phases reduces firstly and then tends to be stable. The change of the angle of the slot does not show effect on the velocity of dispersed phase flow into the cyclone reactor. But when the angle is too small, the wall adhesion is harmful to the process that dispersed phase flows from cushion chamber into the cyclone reactor. Thereby, when the angle changes from $5^{\circ}$ to $7^{\circ}$, the mean mixingseparation time reduces because of effect of the wall adhesion decreases. Besides, when the angle is greater than $7^{\circ}$, wall adhesion shows little influence on the flow process of dispersed phase from cushion chamber into the cyclone reactor.

4.3.2. Effect of $\boldsymbol{l}$ on the mixing-separation time between two phases. Fig. 10 shows the effect of the length of the slot on the RTD curves at monitoring surface $2^{\#}$ and $3^{\#}$. As shown in Fig. 10a, with the increase of the length of the slot, the peak of the RTD curves at monitoring surface $3^{\#}$ becomes lower and the peak moves to the right, which mean that the flow time from inlet to the monitoring surface $3^{\#}$ increases. Compared with the variation of the RTD curves at monitoring surface $3^{\#}$, the change of the RTD curves at monitoring surface $2^{\#}$ is more obvious. The peak of the RTD curves also moves to the right with the increase of the length of the slot. Fig. 11 shows the variation of the mean mixing-separation time with the increase of the length of the slot. As shown in Fig. 11, the variation tendency is consistent with the results in Fig. 10. It is known that the flow velocity of two phases all show effect on the mixing-separation time in the cyclone reactor from the introduction of the cyclone reactor. The relative velocity between two phases increases the residence 


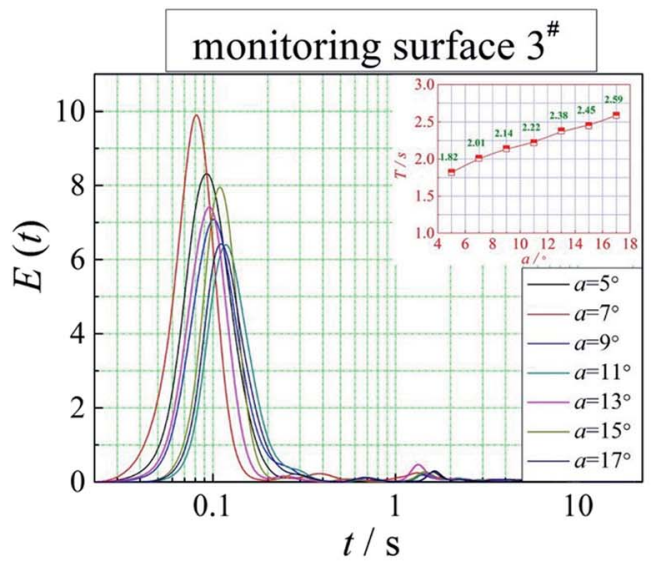

(a)

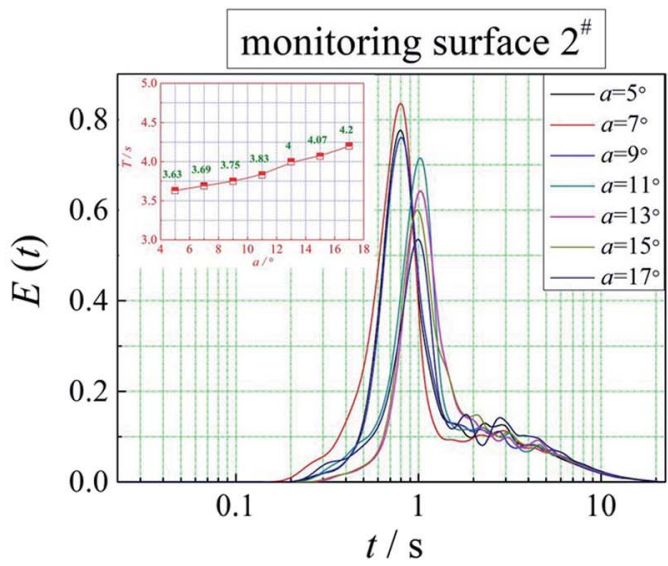

(b)

Fig. 8 Effect of $a$ on the RTD curves

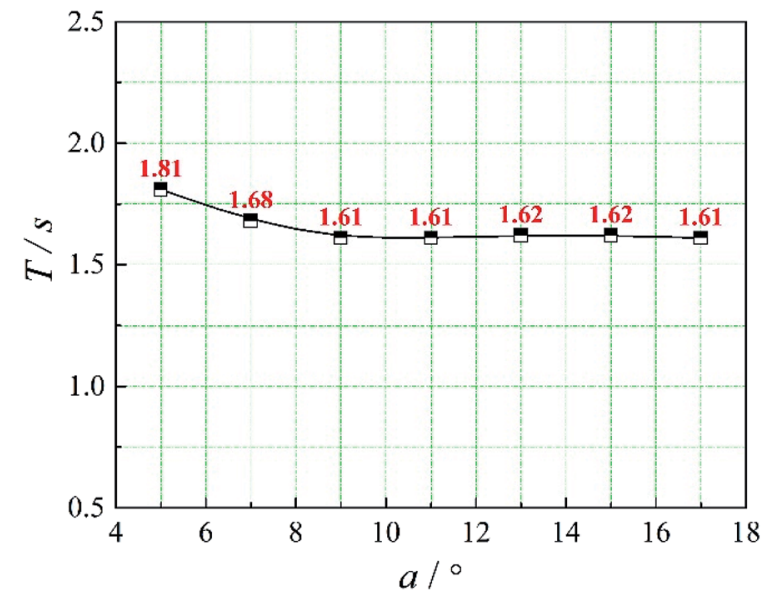

Fig. 9 Mean mixing-separation time under different angles of the slot.

time of dispersed phase in the cyclone reactor. Therefore, the decrease of the velocity of dispersed phase caused by the increase of the length of the slot leads to the increase of the relative velocity between two phases. As a result, the mean mixing-separation time between two phases in the cyclone reactor increases.

4.3.3. Effect of $\boldsymbol{n}$ on the mixing-separation time between two phases. The effect of the number of the slot on the RTD curves at monitoring surface $2^{\#}$ and $3^{\#}$ is shown in Fig. 12. It can be seen that the variation of the RTD curves is not regular with the increase of the number of the slot both at monitoring surface $2^{\#}$ and $3^{\#}$. But the flow time that dispersed phase flow to monitoring surface $2^{\#}$ and $3^{\#}$ changes regularly over the number of the slot. As shown in Fig. 12, the time that dispersed phase flow to monitoring surface $3^{\#}$ decreases with the increase of the number of the slot. And the time that dispersed phase flow to monitoring surface $2^{\#}$ also shows a downward trend with the increase of the number of the slot generally. Fig. 13 shows the variation of the mean mixingseparation time with the increase of the number of the slot.
As shown in Fig. 13, the mean mixing-separation time increase with the increase of the number of the slot. The velocity of dispersed phase flow into the reaction chamber decreases with the increase of the number of the slot. Meanwhile, the time that the dispersed phase stay in the cushion chamber decreases. Therefore, the time that the dispersed phase flow into the monitoring surface $3^{\#}$ was affected by the mentioned two aspects. Besides when the number of the slot is 1, the RTD curves is bimodal at monitoring surface $2^{\#}$ and $3^{\#}$. We all know that the dispersed phase flow from the inlet to the slot in two directions, and the two roads take different time. Thus, there exits two peaks in the RTD of the dispersed phase at monitoring surface $3^{\#}$. Correspondingly, the RTD of the dispersed phase at monitoring surface $2^{\#}$ is bimodal.

4.3.4. Effect of $d$ on the mixing-separation time between two phases. Fig. 14 shows the effect of the distance of the slot on the RTD curves at monitoring surface $2^{\#}$ and $3^{\#}$. As shown in Fig. 14, the influence of the distance on the RTD curves is less than that of the number of the slot, which indicates the variation of the velocity of dispersed phase caused by the distance of the slot is smaller than that caused by the number of the slot. With the increase of the distance of the slot, the time that the dispersed phase flow to the monitoring surface $2^{\#}$ and $3^{\#}$ increases, which is related to that the velocity of dispersed phase decrease with the increase of the distance of the slot. Therefore, the mean mixing-separation time increase with increase of the distance of the slot, which can be seen in Fig. 15. Moreover, the number of the slot is 1 during the investigation of the effect of the distance of the slot on the mean mixingseparation time. Therefore, the RTD curves under different distance of the slot is bimodal, which is consistent with the analysis in 4.3.3.

\subsection{Development prediction model to calculate the mean mixing-separation time}

It can be concluded from the above analysis that the mean mixing-separation time between two phases in the cyclone 


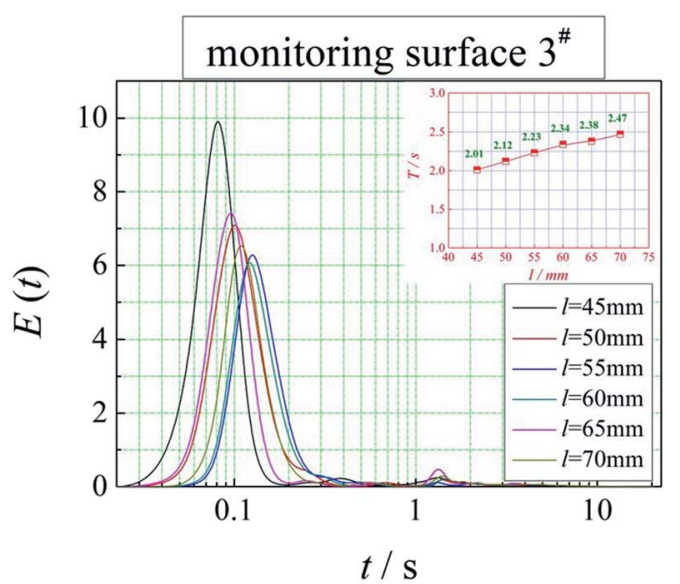

(a)

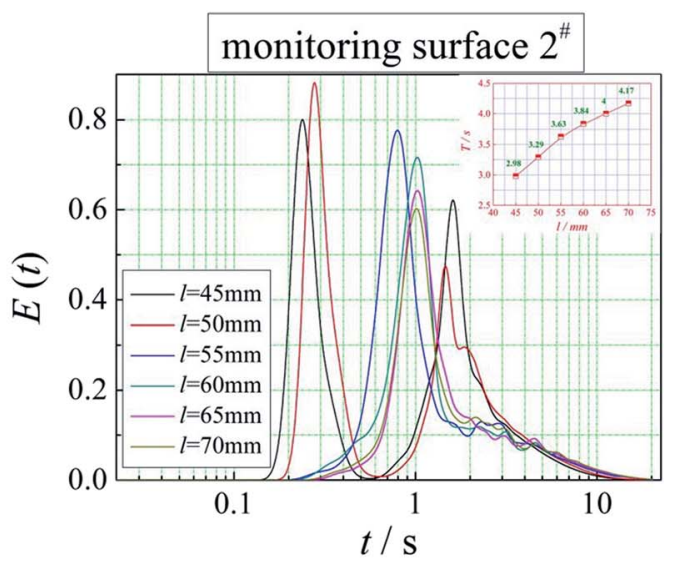

(b)

Fig. 10 Effect of $l$ on the RTD curves.

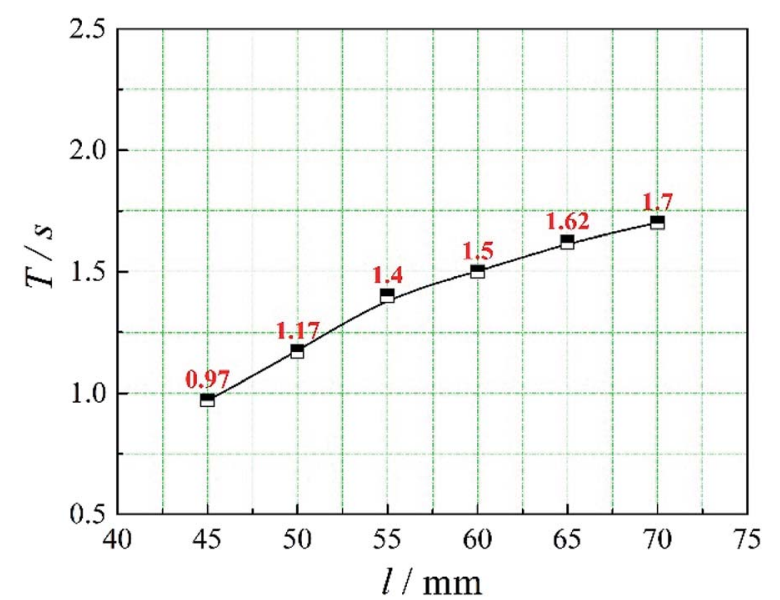

Fig. 11 Mean mixing-separation time under different length of the slot.

reactor, namely the time of the dispersed phase flow from monitoring surface $3^{\#}$ to monitoring surface $2^{\#}$, is related to the velocity of dispersed phase into the reaction chamber.
And the mixing process between two phases in the cyclone reactor is the dispersion of dispersed phase into continuous phase in the form of droplets. Besides, the effect of the operational parameters (total inlet flow, feed ratio, overflow ratio) on the flow field in the cyclone reactor have been investigated in the previous studies. The results show that the total inlet flow and feed ratio are the main influence factors on the flow field in the reaction chamber and the velocities of two phases change with the change of total inlet flow and feed ratio. ${ }^{20,23}$ Based on these results, we can conclude that the relative velocity between two phases plays an important role in the dispersion process. Therefore, we established a prediction model to explain the relationship between the mean mixing-separation time and the velocity of two phases, which is shown in eqn (12).

$$
T=a v_{d}^{b} v_{c}^{c}+d
$$

The 1stopt5.0 software was used in the fitting process to calculate the coefficient in the prediction model. The optimized $a, b, c, d$ is $-0.137,0.135,-67.767,9.174$, respectively.

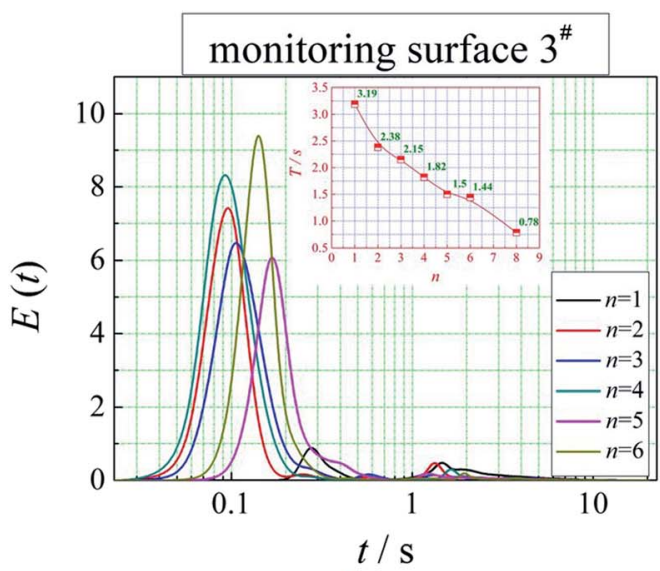

(a)

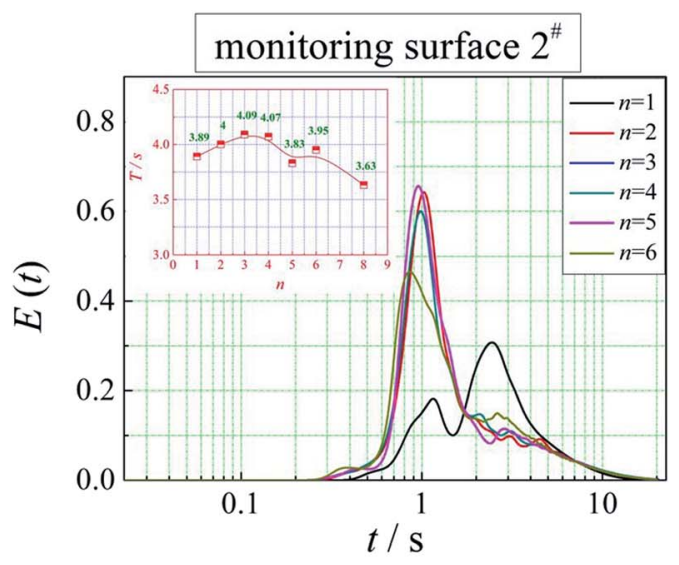

(b)

Fig. 12 Effect of $n$ on the RTD curves. 


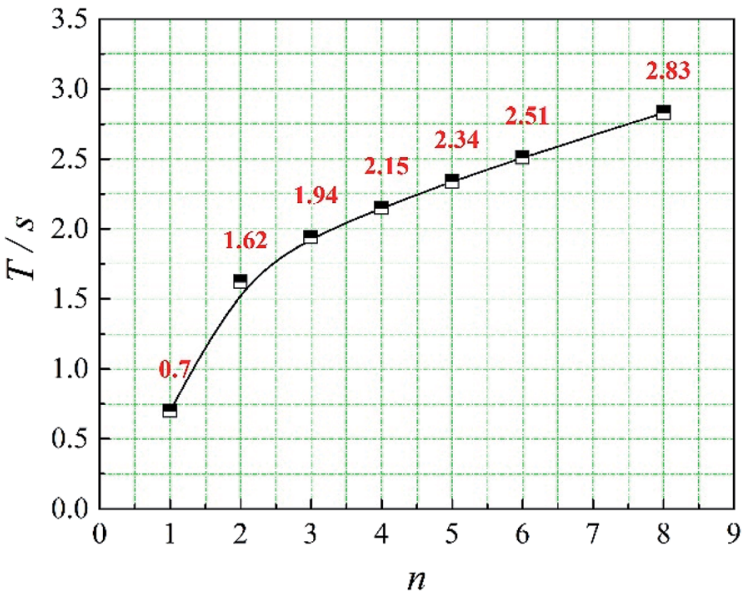

Fig. 13 Mean mixing-separation time under different length of the slot.

Therefore, the relationship between the mean mixingseparation time and velocity of two phases is as shown in eqn (11). Moreover, the comparison between the prediction data and the simulated data is shown in Fig. 16. As shown in Fig. 16, the prediction data agrees well with the simulated data.

$$
T=-0.137 v_{d}^{0.135} v_{c}{ }^{-67.767}+9.174
$$

Moreover, if the basic hypothesis of the prediction model is reasonable, the standardized residual, $e_{\text {is }}$, obeys a normal distribution, therefore,

$$
P\left\{\left|e_{\text {is }}\right|<2\right\} \approx 0.95
$$

The distribution of $e_{\text {is }}$ is shown in Fig. 7. As shown in Fig. 17, more than $95 \%$ of the points are in the belt area $\left(e_{\text {is }} \pm 2\right)$ with

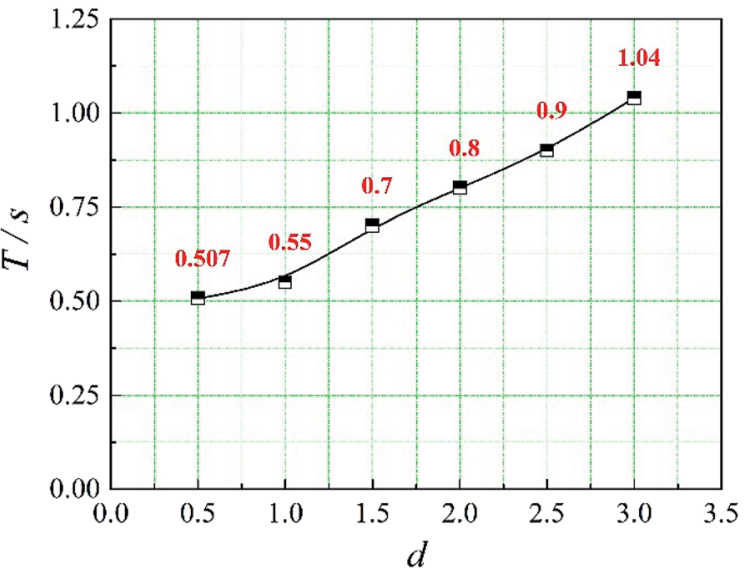

Fig. 15 Mean mixing-separation time under different distance of the slot.

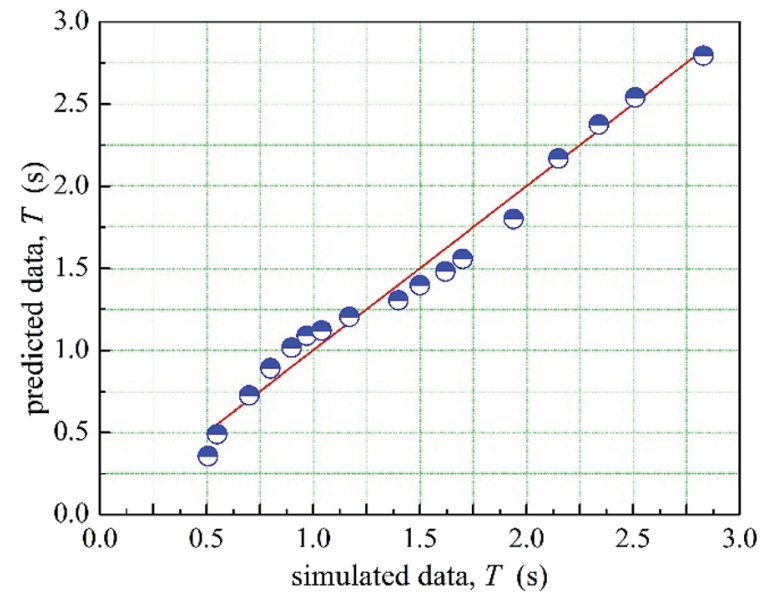

Fig. 16 Comparison between fitting data and simulated data.

a random distribution, which indicated the predicted data and experimental data are in good agreement. Therefore, the prediction model can be used to calculate the mean mixingseparation time between two phases in the cyclone reactor.

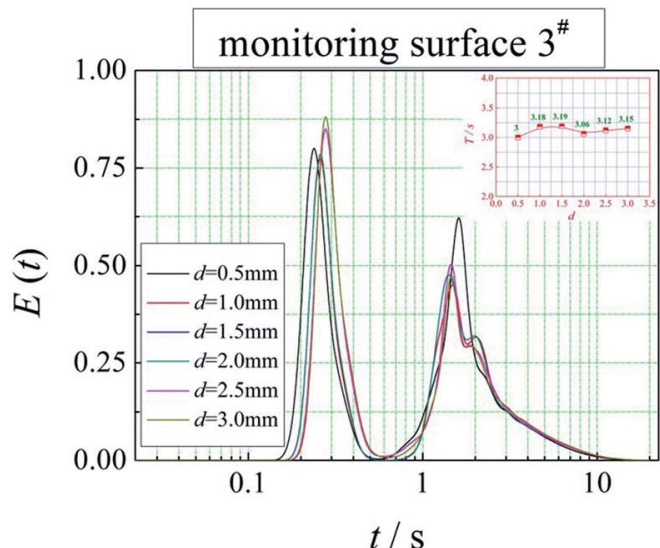

(a)

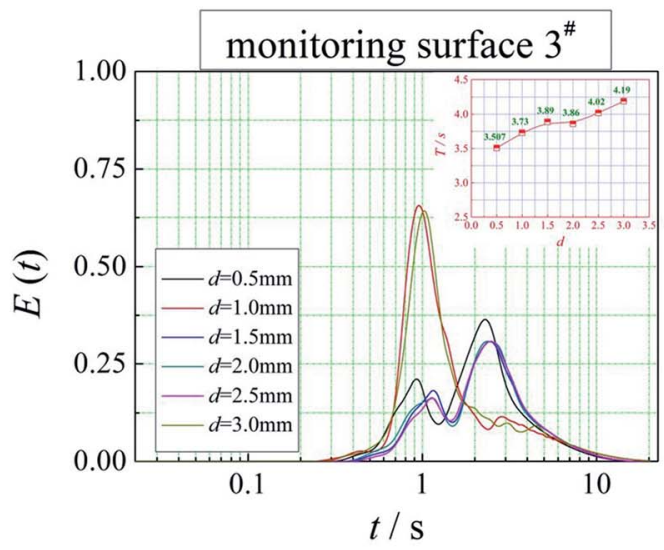

(b)

Fig. 14 Effect of $d$ on the RTD curves. 


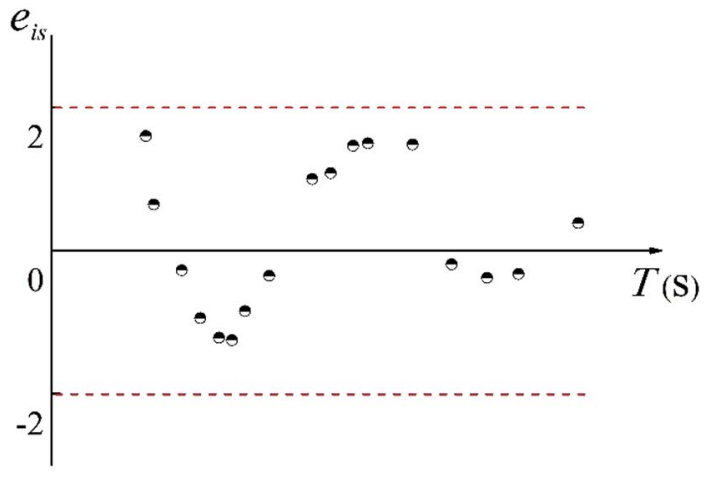

Fig. 17 Residual graph.

\section{Conclusion}

In this paper, a CFD model was adopted to investigate the mixing-separation time between two phases in a multi-element process coupled cyclone reactor for ionic liquid-catalyzed isobutane/butene alkylation. We investigated the effect of structural parameters of the dispersed phase inlet (the slot) on the residence time distributions of dispersed phase and mean mixing-separation time between two phases in the cyclone reactor. The results show that the main factor to affect the mean mixing-separation time are the velocity of dispersed phase flow into the reaction chamber and the relative velocity between two phases in the cyclone reactor. Besides, when the number of the slot is 1 , the residence time distribution is a bimodal distribution. Moreover, a prediction model to calculate the mean mixing-separation time between two phases in the cyclone reactor was established.

\section{Conflicts of interest}

There are no conflicts to declare.

\section{Acknowledgements}

The authors gratefully acknowledge the support from the National Science Fund for Distinguished Young Scholars: No. 21425626, National Natural Science Foundation of China: No. 51806248 and the Doctoral Research Fund of Shandong Jianzhu University: No. X18068Z.

\section{References}

1 W. Z. Zheng, H. Y. Wang, W. X. Xie, L. Zhao and W. Z. Sun, AIChE J., 2017, 64(3), 950-960.

2 H. Wang, X. Z. Meng, G. Y. Zhao and S. J. Zhang, Green Chem., 2017, 19(6), 1462-1489.

3 L. F. Albright, Ind. Eng. Chem. Res., 2009, 48(3), 1409-1413. 4 J. S. Wilkes, J. A. Levisky, R. A. Wilson and C. Hussey, Inorg. Chem., 1982, 21(3), 1263-1264.
5 T. Welton, Chem. Rev., 1999, 99(8), 2071-2084.

6 R. Sheldon, Chem. Commun., 2001, 23, 2399-2407.

7 J. F. Brennecke and E. J. Maginn, AIChE J., 2001, 47(11), 2384-2389.

8 K. Yoo, V. V. Namboodiri, R. S. Varma and P. G. Smirniotis, J. Catal., 2004, 222, 511-519.

9 L. Schmerling, J. Am. Chem. Soc., 2002, 68(2), 2491-2497.

10 J. J. Zhou, Z. C. Liu and M. X. Liu, Acta Pet. Sin., Pet. Process. Sect., 2008, 24(2), 134-140.

11 S. J. Royaee, M. Sohrabi and A. Shafeghat, Korean J. Chem. Eng., 2014, 31(2), 240-247.

12 J. Lédé, F. Broust, F. T. Ndiaye and M. Ferrer, Fuel, 2007, 86(12-13), 1800-1810.

13 V. Shilapuram, D. Jaya Krishna and N. Ozalp, Int. J. Hydrogen Energy, 2011, 36(21), 13488-13500.

14 A. M. Fonseca, J. J. Órfão and R. L. Salcedo, Ind. Eng. Chem. Res., 2001, 40(1), 304-313.

15 D. Weuster-Botz, E. Hünnekes and A. Hartbrich, Bioprocess Eng., 1998, 18(6), 433.

16 Y. C. Zhang, Z. B. Wang and Y. H. Jin, Chem. Eng. Res. Des., 2013, 91(9), 1768-1776.

17 P. V. Danckwerts, Chem. Eng. Sci., 1953, 2(1), 1-13.

18 I. L. Gamba, S. Marquez Damian, D. A. Estenoz, N. Nigro, M. A. Storti and D. Knoeppel, Int. J. Chem. React. Eng., 2012, 10(1), 1542-6580.

19 J. T. Adeosun and A. Lawal, Chem. Eng. Sci., 2009, 64(10), 2422-2432.

20 M. Y. Zhang, L. Wang, L. Y. Zhu, Z. B. Wang, Z. C. Liu and Y. H. Jin, Chem. Eng. Res. Des., 2017, 125, 257-264.

21 Z. B. Wang, M. Y. Zhang, Z. C. Liu, L. Y. Zhu, Y. H. Jin and C. M. Xu, Chem. Eng. Res. Des., 2018, 137, 502-509.

22 M. Y. Zhang, T. Y. Zhang, L. Y. Zhu, Z. B. Wang and Y. H. Jin, Powder Technol., 2017, 316, 289-295.

23 M. Y. Zhang, L. Y. Zhu, Z. B. Wang, Z. C. Liu, Z. Z. Liu, C. M. Xu and Y. H. Jin, Chem. Eng. Res. Des., 2017, 125, 282-290.

24 Z. B. Wang, Y. Ma and Y. H. Jin, Chem. Eng. Res. Des., 2011, 89(6), 603-610.

25 M. Narasimaha, R. Sripriya and P. K. Banerjee, Int. J. Miner. Process., 2005, 75(1-2), 53-68.

26 T. B. Anderson and R. A. Jackson, Ind. Eng. Chem. Fundam., 1967, 6, 527-534.

27 R. M. Bowen, in Continuum Physics, ed. A. C. Eringen, Academic Press, New York, 1967, pp. 1-127.

28 A. Tomiyama, H. Tamai, I. Zun and S. Hosokawa, Chem. Eng. Sci., 2002, 57(11), 1849-1858.

29 D. A. Drew and R. T. Lahey, in Particulate Two-Phase Flow, Butterworth-Heinemann, Boston, 1993, pp. MA509-566.

30 M. Y. Zhang, A. J. Li, L. Y. Zhu, Z. B. Wang, Z. C. Liu and Y. H. Jin, Separation and Purification Technology, 2019, 209, 375-382. 